\title{
Prophylactic administration of mecapegfilgrastim after chemotherapy in patients with lymphoma
}

\author{
Meijing Zheng", Xiaolian Wen", Liping Su \\ Department of Hematology, Affiliated Shanxi Tumor Hospital of Shanxi Medical University, Taiyuan, China \\ Contributions: (I) Conception and design: X Wen; (II) Administrative support: L Su; (III) Provision of study materials or patients: L Su; (IV) \\ Collection and assembly of data: M Zheng; (V) Data analysis and interpretation: M Zheng; (VI) Manuscript writing: All authors; (VII) Final approval \\ of manuscript: All authors. \\ "These authors contributed equally to this work. \\ Correspondence to: Liping Su. Department of Hematology, Affiliated Shanxi Tumor Hospital of Shanxi Medical University, Taiyuan 030013, China. \\ Email: sulp2005@163.com.
}

Background Neutropenia is a common and serious complication encountered during chemotherapy
treatment of cancer patients. The incidence of neutropenia increases the risk of infection and can influence
the chemotherapy treatment in terms of drug dosage and treatment duration. Mecapegfilgrastim is a
novel, long-acting pegylated recombinant human granulocyte-colony stimulating factor (PEG-rhG-CSF)
designed to prevent the incidence of neutropenia. The study aims to observe the effectiveness and safety of
mecapegfilgrastim as prophylaxis for chemotherapy-induced neutropenia in patients with lymphoma. Methods: Ninety-one patients with lymphoma were enrolled and received mecapegfilgrastim as either primary or secondary prophylaxis. The incidence of grade III/IV neutropenia, the duration of grade III/IV neutropenia in the overall population, and the differences between the primary and secondary prophylaxis groups were investigated. Adverse events were also recorded.

Results: During the first chemotherapy cycle, the incidence of grade III and grade IV neutropenia was $5 \%$ and $7 \%$, respectively. Of the 71 patients who received mecapegfilgrastim as primary prophylaxis, the incidence of grade III and grade IV neutropenia was $4 \%$ and $1 \%$, respectively. Of the 20 patients who received mecapegfilgrastim as secondary prophylaxis, the incidence of grade III and grade IV neutropenia was $10 \%$ and $25 \%$, respectively. The mean duration of grade III neutropenia was 0.85 days. The mean duration of grade III neutropenia in patients who received mecapegfilgrastim as primary prophylaxis was one day less than patients who received mecapegfilgrastim as secondary prophylaxis. Fever and bone/muscle pain were the most frequently observed adverse events.

Conclusions: Mecapegfilgrastim is more effective in reducing the incidence of grade III/IV neutropenia and the mean duration of febrile neutropenia $(\mathrm{FN})$ when used as primary prophylaxis rather than secondary prophylaxis in patients with lymphoma. The toxicity of mecapegfilgrastim was tolerable.

Keywords: Mecapegfilgrastim; lymphoma; neutropenia

Submitted Mar 01, 2021. Accepted for publication Dec 23, 2021.

doi: 10.21037/apm-21-3209

View this article at: https://dx.doi.org/10.21037/apm-21-3209

\section{Introduction}

Lymphoma is the most common form of hematological malignancy. The two main types of lymphoma are Hodgkin lymphoma (HL) and non-Hodgkin lymphoma (NHL).
Lymphoma, with a death rate of 36.2 per million people, was the tenth most fatal cancer in China in 2015 (1). Lymphoma is a systemic disease. Except for those with limited lesions (early stage/stage I) who choose radiotherapy or surgery for treatment, most patients should first receive 
systemic chemotherapy.

Among cancer patients receiving myelosuppressive chemotherapy, febrile neutropenia $(\mathrm{FN})$ is a potentially life-threatening complication (2-4). FN usually leads to a longer hospital stay, an increase in treatment costs, and a reduction or delay in the dose of chemotherapy, thereby affecting the quality of life of the patient and increasing the risk of death (5-7). Prophylactic use of granulocytecolony stimulating factor (G-CSF) for patients with myelosuppressive chemotherapy can reduce the incidence of FN, agranulocytosis, and infection (8-11).

Mecapegfilgrastim is a long-acting pegylated recombinant human granulocyte-colony stimulating factor (PEG-rhGCSF) which has been developed to reduce the incidence of FN. Mecapegfilgratim only needs to be administered once per chemotherapy cycle. This is more convenient than short-acting G-CSF and will elevate the compliance of patients receiving chemotherapy. The efficacy and safety of mecapegfilgrastim in breast cancer and non-small cell lung cancer patients has been studied in two pivotal trials $(12,13)$. Compared with short acting G-CSF (filgrastim), mecapegfilgrastim was superior on reducing duration of grade $\geq 3$ neutropenia in breast cancer patients treated with chemotherapy (14). However, research on the use of mecapegfilgrastim in lymphoma patients is lacking.

We present the following article in accordance with the TREND reporting checklist (available at https://dx.doi. org/10.21037/apm-21-3209).

\section{Methods}

All procedures performed in this study involving human participants were in accordance with the Declaration of Helsinki (as revised in 2013). This study was registered on Chinese Clinical Trial Registry (https://www.chictr.org. cn/index.aspx) (No.: ChiCTR2100048123). The study was approved by Chinese Ethics Committee of Registering Clinical Trials (No.: ChiECRCT20210350). All patients signed informed consent forms.

\section{Eligibility criteria}

Patients with HL or NHL from Shanxi Tumor Hospital who received at least one cycle of chemotherapy were enrolled. The inclusion criteria were as follows: age $\geq 18$ years; the ability to complete at least one cycle of chemotherapy as planned (patients with an intermediate risk of FN needed to have more than one risk factor); a normal bone marrow hematopoietic function with no bleeding tendency; absolute neutrophil count (ANC) $\geq 2.0 \times 10^{9} / \mathrm{L}$, serum alanine aminotransferase (ALT) and aspartate aminotransferase (AST) $\leq 1.5$ times of upper limits of normal (ULN), serum total bilirubin (TBIL) $\leq 1.5$ times of ULN, and serum creatinine (Scr) $\leq 1.5$ times of ULN. The exclusion criteria were as follows: presence of acute infection and receipt of systemic anti-infection treatment in the 72 hours before chemotherapy; presence of hematological diseases affecting the hematopoietic function of bone marrow; patients who had bone marrow or stem-cell transplantation within the past 3 months; presence of other uncured or brain metastatic malignant tumors; allergic or intolerable to the study drugs; presence of mental or nervous system disorders; women who were pregnant or breast feeding; women of childbearing age who refused contraceptive use; patients who had previously received PEG-rhG-CSF; and some special cases that the investigators determined should be excluded.

\section{Mecapegfilgrastim administration}

Primary prophylaxis: In accordance with the National Comprehensive Cancer Network (NCCN) guidelines (NCCN Clinical Practice Guidelines in Oncology (NCCN Guidelines ${ }^{\circledR}$ ) for Hematopoietic Growth Factors, version 2.2019), G-CSF was administered to patients with a higher than $20 \%$ risk of developing $\mathrm{FN}$ after the first chemotherapy cycle; for patients with an overall risk of developing FN between $10 \%$ and $20 \%$, G-CSF was considered after evaluation of patient risk factors.

Secondary prophylaxis: An incidence of FN or a doselimiting neutropenia event in the previous chemotherapy cycle suggested administration of G-CSF in the next chemotherapy cycle.

Eligible patients were given mecapegfilgrastim (fixed dose, $6 \mathrm{mg}) 48 \mathrm{~h}$ after chemotherapy by a single subcutaneous injection.

\section{Effectiveness assessment}

The primary endpoint was the incidence of grade III/ IV neutropenia (ANC $<1.0 \times 10^{9} / \mathrm{L}$ ) in the first cycle after mecapegfilgrastim administration. The secondary efficacy endpoints included the mean duration of grade III/IV neutropenia in the first cycle after mecapegfilgrastim administration; the incidence of grade III/IV neutropenia in patients receiving primary or secondary prophylaxis; and 
Table 1 Baseline characteristics of patients

\begin{tabular}{lc}
\hline Characteristics & $\mathrm{n}(\%)$ \\
\hline Age group & $22(24.2)$ \\
18-65 & $69(75.8)$ \\
$>65$ & \\
Sex & $54(59.3)$ \\
Male & $37(40.7)$ \\
Female & \\
The Karnofsky performance scale & $2(2.2)$ \\
60 & $13(14.3)$ \\
70 & $71(78.0)$ \\
80 & $5(5.5)$ \\
90 & \\
Tumor type & $22(24.2)$ \\
Diffused large B-cell lymphoma & $41(45.0)$ \\
T-cell lymphoma & $15(16.5)$ \\
B-cell lymphoma & $3(3.3)$ \\
Other non-Hodgkin's lymphoma & \\
Classic Hodgkin's lymphoma & $11(12.1)$ \\
III & \\
\hline
\end{tabular}

the mean duration of grade III/IV neutropenia in patients receiving primary or secondary prophylaxis.

\section{Safety assessment}

Adverse events in eligible patients were recorded.

\section{Statistical analysis}

Descriptive statistics were used to summarize the baseline characteristics, effectiveness, and safety data. All statistical analysis was applied with SPSS 17.0. Categorial variables were presented as number and percentage, $\mathrm{n}(\%)$. Continuous variables were classified into different groups and presented as $\mathrm{n}(\%)$. Adverse events were recorded and presented as $\mathrm{n}(\%)$.

\section{Results}

\section{Patients}

From January 2018 to September 2019, 91 eligible patients were administered mecapegfilgrastim after a single cycle of chemotherapy. Sixty-nine (76\%) patients were older than 65 years. Fifty-four (59\%) were male patients. Seventy-one (78\%) patients had Karnofsky Performance Scale (KPS) scores of 80 . Forty-nine (54\%) patients had Diffuse large B-Cell lymphoma. Sixty-two (67\%) patients were classified as phase III/IV lymphoma (Table 1).

\section{Effectiveness}

During the first chemotherapy cycle, $11(12 \%)$ patients had grade III/IV neutropenia. The mean duration of grade III/ IV neutropenia was 0.85 days.

In 71 patients receiving primary prophylaxis, the incidence of grade III and grade IV neutropenia was $4 \%$ and $1 \%$, respectively. The mean duration of grade III/IV neutropenia of these patients was 0.63 days (Table 2).

In 20 patients receiving secondary prophylaxis, the incidence of grade III and grade IV neutropenia was $10 \%$ and $25 \%$, respectively. The mean duration of grade III/IV neutropenia of these patients was 1.6 days (Table 2).

\section{Safety}

Of the total 91 patients who received mecapegfilgrastim, $9(9.9 \%)$ and 6 (6.6 \%) patients reported fever and bone/ muscle pain, respectively. Nausea, vomiting, and malaise were observed in $3(3 \%), 3(3 \%)$, and $3(3 \%)$ patients, respectively. The observed adverse events were mostly grade I or grade II. No adverse event greater than grade III was observed. The detailed adverse events are presented in Table 3. Of all the adverse events, the occurrence of bone/ muscle pain was considered very likely related to the investigated drug; Fever, nausea, vomiting, and malaise were likely associated with the investigated drug; and pruritus, dyspnea and skin ulceration might not be related to the investigated drug.

\section{Discussion}

This study presented the effectiveness and safety of mecapegfilgrastim administration in lymphoma patients in a real-world setting. To our knowledge, this is the first clinical study of mecapegfilgrastim in this circumstance. The results 
Table 2 Effectiveness results in cycle one

\begin{tabular}{|c|c|c|c|}
\hline Variable & Primary prophylaxis, $\mathrm{N}=71$ & Secondary prophylaxis, $\mathrm{N}=20$ & All, $\mathrm{N}=91$ \\
\hline Grade IV neutropenia, n (\%) & $1(1.4)$ & $5(25.0)$ & $6(6.6)$ \\
\hline Mean duration of grade III/IV neutropenia, days & 0.63 & 1.60 & 0.85 \\
\hline
\end{tabular}

Table 3 Adverse events

\begin{tabular}{ll}
\hline Adverse events & $\mathrm{n}(\%)$ \\
\hline Fever & $9(9.9)$ \\
Bone/muscle pain & $6(6.6)$ \\
Nausea & $3(3.3)$ \\
Vomiting & $3(3.3)$ \\
Malaise & $3(3.3)$ \\
Pruritus & $1(1.1)$ \\
Dyspnea & $1(1.1)$ \\
Skin ulceration & $1(1.1)$ \\
\hline
\end{tabular}

of this study indicated that prophylactic administration of mecapegfilgrastim in lymphoma patients resulted in an incidence of grade III/IV neutropenia of about $12 \%$. This is lower than the incidence rates in breast cancer $(51 \%)$ and advanced non-small cell lung cancer (14\%) patients in the pivotal trials $(12,14)$. The mean duration of grade III/IV neutropenia in patients with lymphoma was 0.85 days which is shorter than that of breast cancer patients (1.23 days) and longer than that of advanced non-small cell lung cancer patients $(0.69$ days $)$ in the pivotal trials $(12,14)$.

More than $50 \%$ of breast cancer patients experienced grade III or grade IV neutropenia following administration of mecapegfilgrastim in cycle one in the phase 3 study of mecapegfilgrastim (14). In this study, we did not find as high an incidence of neutropenia in cycle one as in the previous phase 3 breast cancer trial. This probably resulted from the chemotherapy (AT, anthracyclines-taxane or AC, adriamycin and

cyclophosphamide) used for the breast cancer patients at that time. The chemotherapy used for lymphoma patients in this study was more diverse and of lower toxicity.

Primary prophylaxis showed a reduction in the incidence of grade III/IV neutropenia and a shorter duration of grade III/IV neutropenia compared with secondary prophylaxis. The safety profile of mecapegfilgrastim administration in lymphoma patients was similar to the safety findings in two pivotal trials. Bone/muscle pain and fever were the most frequently observed adverse events. No serious adverse events have been observed in this study.

Primary or secondary prophylaxis of neutropenia using G-CSF has been investigated in several studies. A study conducted in Belgium and Luxembourg indicated that patients who received long-acting G-CSF as primary prophylaxis had a lower incidence of grade III and grade IV neutropenia than those who received secondary administration (15). Another prospective non-interventional study in Germany also reported that primary administration of long-acting G-CSF had a lower incidence of severe neutropenia compared with secondary administration (16). These findings are in line with our findings in this study. Due to the diverse chemotherapy regimen for patients with lymphoma, the optimize administration schedule and dosage of G-CSF still need to explore in large prospective studies.

There were limitations in this study. This study was conducted with a relatively small-sized population. A larger sample size real-world study is warranted to confirm the validity of the present results. Besides, the follow up time was relatively short. A long-term follow-up of patients would provide valuable information on health-related outcome.

\section{Acknowledgments}

Funding: None.

\section{Footnote}

Reporting Checklist: The authors have completed the TREND reporting checklist. Available at https://dx.doi. org/10.21037/apm-21-3209

Data Sharing Statement: Available at https://dx.doi. org/10.21037/apm-21-3209 
Conflicts of Interest: All authors have completed the ICMJE uniform disclosure form (available at https://dx.doi. org/10.21037/apm-21-3209). The authors have no conflicts of interest to declare.

Ethical Statement: The authors are accountable for all aspects of the work in ensuring that questions related to the accuracy or integrity of any part of the work are appropriately investigated and resolved. All procedures performed in this study involving human participants were in accordance with the Declaration of Helsinki (as revised in 2013). This study was registered on Chinese Clinical Trial Registry (No.: ChiCTR2100048123). The study was approved by Chinese Ethics Committee of Registering Clinical Trials (No.: ChiECRCT20210350). All patients signed informed consent forms.

Open Access Statement: This is an Open Access article distributed in accordance with the Creative Commons Attribution-NonCommercial-NoDerivs 4.0 International License (CC BY-NC-ND 4.0), which permits the noncommercial replication and distribution of the article with the strict proviso that no changes or edits are made and the original work is properly cited (including links to both the formal publication through the relevant DOI and the license). See: https://creativecommons.org/licenses/by-nc-nd/4.0/.

\section{References}

1. Zheng RS, Sun KX, Zhang SW, et al. Report of cancer epidemiology in China, 2015. Zhonghua Zhong Liu Za Zhi 2019;41:19-28.

2. Lalami $Y$, Paesmans $M$, Aoun $M$, et al. A prospective randomised evaluation of G-CSF or G-CSF plus oral antibiotics in chemotherapy-treated patients at high risk of developing febrile neutropenia. Support Care Cancer 2004;12:725-30.

3. Kuderer NM, Dale DC, Crawford J, et al. Impact of primary prophylaxis with granulocyte colony-stimulating factor on febrile neutropenia and mortality in adult cancer patients receiving chemotherapy: a systematic review. J Clin Oncol 2007;25:3158-67.

4. Zeng F, Li T, Xia C, et al. Efficacy of Joungal in preventing febrile neutropenia induced by platinum-based doublet chemotherapy in lung cancer. Ann Palliat Med 2020;9:1688-95

5. Smith TJ, Bohlke K, Lyman GH, et al. Recommendations for the Use of WBC Growth Factors: American Society of
Clinical Oncology Clinical Practice Guideline Update. J Clin Oncol 2015;33:3199-212.

6. Crawford J, Becker PS, Armitage JO, et al. Myeloid growth factors, version 2.2017. J Natl Compr Canc Netw 2017;15:1520-41.

7. Aapro MS, Bohlius J, Cameron DA, et al. 2010 update of EORTC guidelines for the use of granulocytecolony stimulating factor to reduce the incidence of chemotherapy-induced febrile neutropenia in adult patients with lymphoproliferative disorders and solid tumours. Eur J Cancer 2011;47:8-32.

8. Vogel CL, Wojtukiewicz MZ, Carroll RR, et al. First and subsequent cycle use of pegfilgrastim prevents febrile neutropenia in patients with breast cancer: a multicenter, double-blind, placebo-controlled phase III study. J Clin Oncol 2005;23:1178-84.

9. Hecht JR, Pillai M, Gollard R, et al. A randomized, placebo-controlled phase ii study evaluating the reduction of neutropenia and febrile neutropenia in patients with colorectal cancer receiving pegfilgrastim with every-2week chemotherapy. Clin Colorectal Cancer 2010;9:95-101.

10. Green MD, Koelbl H, Baselga J, et al. A randomized double-blind multicenter phase III study of fixed-dose single-administration pegfilgrastim versus daily filgrastim in patients receiving myelosuppressive chemotherapy. Ann Oncol 2003;14:29-35.

11. Dale D. Current management of chemotherapy-induced neutropenia: the role of colony-stimulating factors. Semin Oncol 2003;30:3-9.

12. Zhou C, Huang Y, Wang D, et al. A Randomized Multicenter Phase III Study of Single Administration of Mecapegfilgrastim (HHPG-19K), a Pegfilgrastim Biosimilar, for Prophylaxis of Chemotherapy-Induced Neutropenia in Patients With Advanced Non-SmallCell Lung Cancer (NSCLC). Clin Lung Cancer 2016;17:119-27.

13. Wang T, Wu B, Hu X, et al. A randomized multicenter phase II trial of mecapegfilgrastim single administration versus granulocyte colony-stimulating growth factor on treating chemotherapy-induced neutropenia in breast cancer patients. Ann Transl Med 2019;7:196.

14. Xu F, Zhang Y, Miao Z, et al. Efficacy and safety of mecapegfilgrastim for prophylaxis of chemotherapyinduced neutropenia in patients with breast cancer: a randomized, multicenter, active-controlled phase III trial. Ann Transl Med 2019;7:482.

15. Fontaine C, Claes N, Graas MP, et al. Effect of lipegfilgrastim administration as prophylaxis of 
chemotherapy-induced neutropenia on dose modification and incidence of neutropenic events: real-world evidence from a non-interventional study in Belgium and Luxembourg. Acta Clin Belg 2021;76:10-5.

16. Kurbacher CM, Fietz T, Diel IJ, et al. NADIR: A Non-

Cite this article as: Zheng M, Wen X, Su L. Prophylactic administration of mecapegfilgrastim after chemotherapy in patients with lymphoma. Ann Palliat Med 2021;10(12):1205512060. doi: 10.21037/apm-21-3209
Interventional Study on the Prophylaxis of ChemotherapyInduced Neutropenia Using Lipegfilgrastim - First

Interim Analysis. Oncol Res Treat 2015;38:221-9.

(English Language Editor: D. Fitzgerald) 